\title{
Direct Observation of Magnon Fractionalization in the Quantum Spin Ladder
}

\author{
B. Thielemann, ${ }^{1}$ Ch. Rüegg, ${ }^{2}$ H. M. Rønnow, ${ }^{3}$ A. M. Läuchli, ${ }^{4}$ J.-S. Caux, ${ }^{5}$ B. Normand, ${ }^{6}$ D. Biner, ${ }^{7}$ K. W. Krämer, ${ }^{7}$ \\ H.-U. Güdel, ${ }^{7}$ J. Stahn, ${ }^{1}$ K. Habicht, ${ }^{8}$ K. Kiefer, ${ }^{8}$ M. Boehm, ${ }^{9}$ D. F. McMorrow, ${ }^{2}$ and J. Mesot ${ }^{1,3}$ \\ ${ }^{1}$ Laboratory for Neutron Scattering, ETH Zurich and Paul Scherrer Institute, CH-5232 Villigen, Switzerland \\ ${ }^{2}$ London Centre for Nanotechnology and Department of Physics and Astronomy, University College London, \\ London WC1E 6BT, United Kingdom \\ ${ }^{3}$ Laboratory for Quantum Magnetism, Ecole Polytechnique Fédérale de Lausanne, CH-1015 Lausanne, Switzerland \\ ${ }^{4}$ Max Planck Institut für Physik komplexer Systeme, Nöthnitzerstrasse 38, D-01187 Dresden, Germany \\ ${ }^{5}$ Institute for Theoretical Physics, University of Amsterdam, 1018 XE Amsterdam, The Netherlands \\ ${ }^{6}$ Theoretische Physik, ETH-Hönggerberg, CH-8093 Zürich, Switzerland \\ ${ }^{7}$ Department of Chemistry and Biochemistry, University of Bern, CH-3000 Bern 9, Switzerland \\ ${ }^{8}$ BENSC, Helmholtz Centre Berlin for Materials and Energy, D-14109 Berlin, Germany \\ ${ }^{9}$ Institut Laue Langevin, 6 rue Jules Horowitz BP156, 38024 Grenoble CEDEX 9, France
}

(Received 18 December 2008; published 13 March 2009)

\begin{abstract}
We measure by inelastic neutron scattering the spin excitation spectra as a function of applied magnetic field in the quantum spin-ladder material $\left(\mathrm{C}_{5} \mathrm{H}_{12} \mathrm{~N}\right)_{2} \mathrm{CuBr}_{4}$. Discrete magnon modes at low fields in the quantum disordered phase and at high fields in the saturated phase contrast sharply with a spinon continuum at intermediate fields characteristic of the Luttinger-liquid phase. By tuning the magnetic field, we drive the fractionalization of magnons into spinons and, in this deconfined regime, observe both commensurate and incommensurate continua.
\end{abstract}

DOI: 10.1103/PhysRevLett.102.107204

Gapped quantum antiferromagnets (AFs) offer some of the most exotic states of electronic matter ever to be observed, and as such have been the enduring focus of intense theoretical and experimental interest. In an applied magnetic field, the threefold degeneracy of the triplet excitations is lifted and the triplet gap $\Delta$ is closed at a critical field $B_{c}=\Delta / g \mu_{B}$. The properties of the states beyond this quantum critical point (QCP) depend strongly on system dimensionality [1], and in one dimension (1D), where long-range order is forbidden, the field-induced phase is expected to be a spin Luttinger liquid (LL). In this state, spin-flip excitations fractionalize into spinons, elementary $S=1 / 2$ entities, whose excitation spectrum is dramatically different from that of both ordered and truly quantum disordered (QD) magnets.

Here we present the results of inelastic neutron scattering (INS) measurements of the magnetic excitation spectrum of a quantum spin ladder. By tuning the magnetic field, we access the full ladder phase diagram, which includes QD, LL, and fully saturated (FM) phases. INS is the only experimental technique which can measure the full momentum and energy dependence of the dynamical susceptibility, information which is essential to identify unambiguously the nature of the quasiparticles in these different phases. We will show that both the QD phase (ladder magnetization $m=0)$ and the FM phase $(m=1)$ have well-defined magnon modes. In sharp contrast, the spectrum in the gapless LL state $(0<m<1)$ is a continuum, whose spectral weight and incommensurate wave vector we control systematically.

Previous attempts to observe the essential physics of the field-induced LL phase in quasi-1D magnets such as the
PACS numbers: 75.10.Jm, 64.70.Tg, 75.40.Gb, 78.70.Nx

candidate Haldane material NDMAP [2], effective $S=1$ chain IPA- $\mathrm{CuCl}_{3}$ [3], and possible spin-ladder system $\mathrm{CuHpCl}$ [4] have generally encountered problems due to additional terms in the spin Hamiltonian. These include single-ion anisotropy, Dzyaloshinskii-Moriya interactions, and especially interchain couplings. While a spinon continuum has been measured in the gapless chain materials $\mathrm{Cu}$-benzoate [5], $\mathrm{CuPzN}$ [6], and $\mathrm{KCuF}_{3}$ [7], the spinladder compound piperidinium copper bromide $\left[\left(\mathrm{C}_{5} \mathrm{H}_{12} \mathrm{~N}\right)_{2} \mathrm{CuBr}_{4}\right][8,9]$ offers the first opportunity to induce LL physics in a gapped system by an applied magnetic field. A number of thermodynamic measurements, specifically of thermal expansion [10], specific heat [11], and magnetocaloric effect $[11,12]$, as well as by nuclear magnetic resonance [13], are consistent with predictions for an ideal ladder. By INS the rung and leg exchange parameters, $J_{r}$ and $J_{l}$, as well as additional terms in the spin Hamiltonian, are determined directly.

High-quality single crystals of $\left(\mathrm{C}_{5} \mathrm{D}_{12} \mathrm{~N}\right)_{2} \mathrm{CuBr}_{4}$ were grown from solution, and up to 10 were coaligned to obtain samples with masses of approximately $2.5 \mathrm{~g}$. INS experiments were performed on the spectrometers IN14 (ILL, Grenoble), FLEX (HMI, Berlin), and TASP (SINQ, Villigen), using a focusing monochromator/analyzer and a Be filter between sample and analyzer (fixed final energies $E_{f}=3.5 \mathrm{meV}$ or $E_{f}=4.7 \mathrm{meV}$ ). Cryomagnets were used for vertical fields up to $14.8 \mathrm{~T}[B \|(b$ axis $)]$.

INS data in the QD phase, $B<B_{c}$, are summarized in Fig. 1. Here we focus on the 1D ladder dispersion: sharp (resolution-limited) peaks arise from a dispersive triplet excitation for momentum transfers along the ladder $\left[Q_{h}\right.$, Fig. 1(b)]. The dispersion in the perpendicular directions 


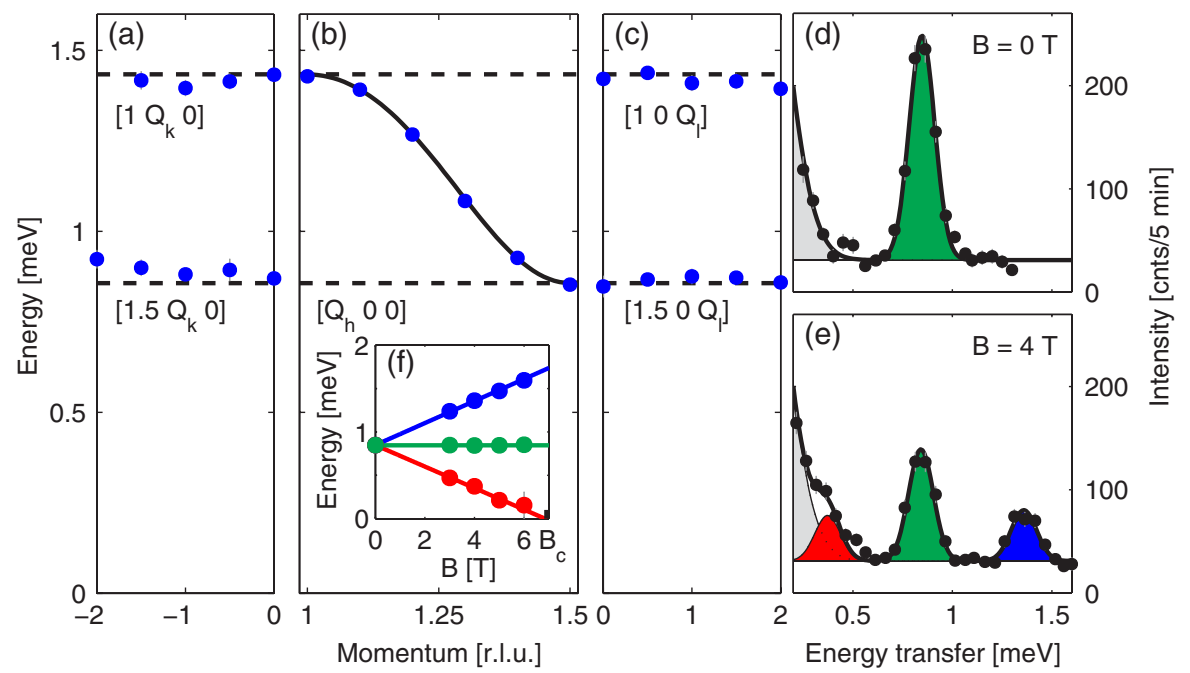

FIG. 1 (color online). Spin dynamics in the QD phase $\left(B<B_{c}\right)$ at $T=$ $50 \mathrm{mK}$. (a)-(c) Triplet dispersion at $B=$ $0 \mathrm{~T}$ from constant- $Q$ scans along the ladder axis and in the two perpendicular directions. (d)-(e) Triplet excitations at $\boldsymbol{Q}=\left[\begin{array}{lll}1.5 & 0 & 0\end{array}\right]$ in fields of 0 and $4 \mathrm{~T}$. (f) Zeeman splitting of the triplet modes at $\boldsymbol{Q}=\left[\begin{array}{lll}1.5 & 0 & 0\end{array}\right]$. Solid lines are fits explained in the text.

[ $Q_{k}$ and $Q_{l}$, Figs. 1(a) and 1(c)] is shown to demonstrate the excellent one-dimensionality of the system. In fact, we have found a systematic variation of order $30 \mu \mathrm{eV}$ in the effective ladder bandwidth [14], and subtract this interladder contribution to obtain the intrinsic parameters $J_{r}$ and $J_{l}$. Figures $1(\mathrm{~d})$ and $1(\mathrm{e})$ show the INS intensity at $\boldsymbol{Q}=$ $\left[\begin{array}{lll}1.5 & 0 & 0\end{array}\right]$ (the band minimum), demonstrating a Zeeman splitting into three triplet components at finite field; Gaussian fits yield the energies shown in Fig. 1(f).

The spectrum changes dramatically above $B_{c}$ : we find a continuum of excitations extending over much of the Brillouin zone and up to energies of $0.8 \mathrm{meV}$. We consider first a field corresponding to half magnetic saturation $(\mathrm{m}=$ 0.5, Fig. 2), where the ladder is equivalent to a gapless spin chain in zero field (below). Here the continuous spectrum of spinon excitations [15] is bounded by $\epsilon_{l}\left(Q_{h}\right)=\hbar \omega\left(Q_{h}\right)$ and $\epsilon_{u}\left(Q_{h}\right)=2 \hbar \omega\left(\frac{Q_{h}}{2}\right)$, where $\hbar \omega\left(Q_{h}\right)=\alpha J\left|\sin \left(2 \pi Q_{h}\right)\right|$ [16] with $\alpha$ a quantum renormalization factor which is determined exactly from the system geometry and interaction parameters. In Fig. 2(a), it is clear that the commensurate $m=0.5$ spectrum is well described by such a shape. Its continuum nature is illustrated strikingly in high-statistics measurements taken at $B=10.1 \mathrm{~T}$ : both constant- $E$ [Figs. 2(b) and 2(d)] and constant- $Q$ [Fig. 2(c)] scans show broad regions of continuous intensity, the latter extending from $0.15 \mathrm{meV}$ (lower measurement limit) to $0.8 \mathrm{meV}$. We stress two important points. First, these data are taken well inside the LL regime, at a temperature significantly below the LL crossover, $T_{\mathrm{LL}}$ [11], but above the boundary to 3D order induced by
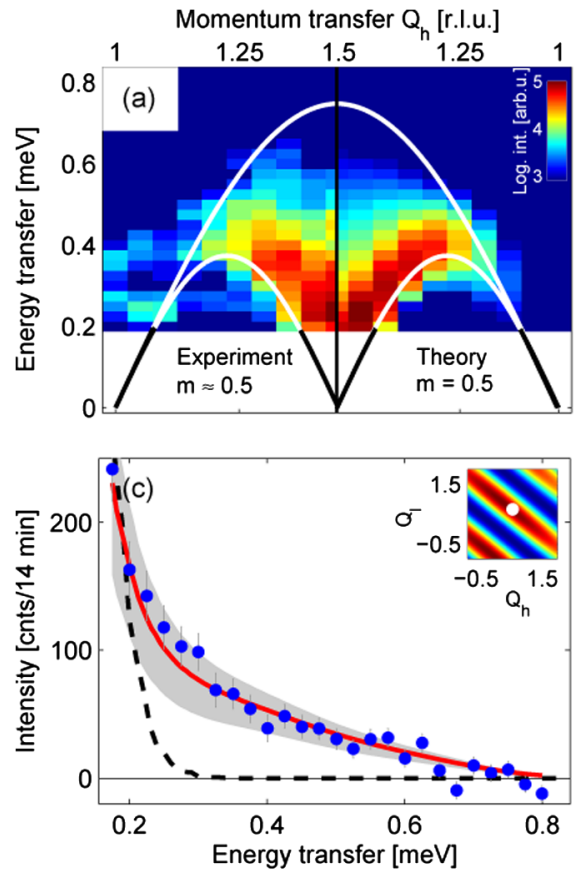
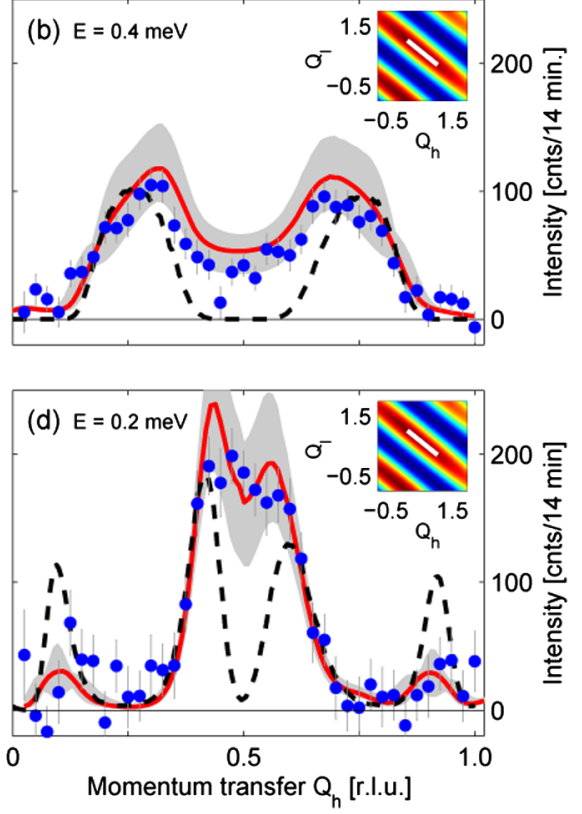

FIG. 2 (color online). Excitation spectrum in the LL phase $\left(T_{N}<T=\right.$ $\left.250 \mathrm{mK}<T_{\mathrm{LL}}\right)$ at $B=10.1 \mathrm{~T} \quad(m \approx$ $0.5)$ after subtraction of the zero-field background. (a) Measured (left) and simulated (right) INS intensities. Solid lines mark the edges of the two-spinon continuum. (b),(d) Constant- $E$ scans taken along maxima of the transverse structure factor (insets: scan trajectories in white). (c) Constant- $Q$ scan at $Q=$ [0.5 0 0.61]. Black dashed lines in (b)(d) are based on a $\delta$-function spinon spectrum [solid lines in panel (a)], red solid lines and shading on a full continuum calculation. 

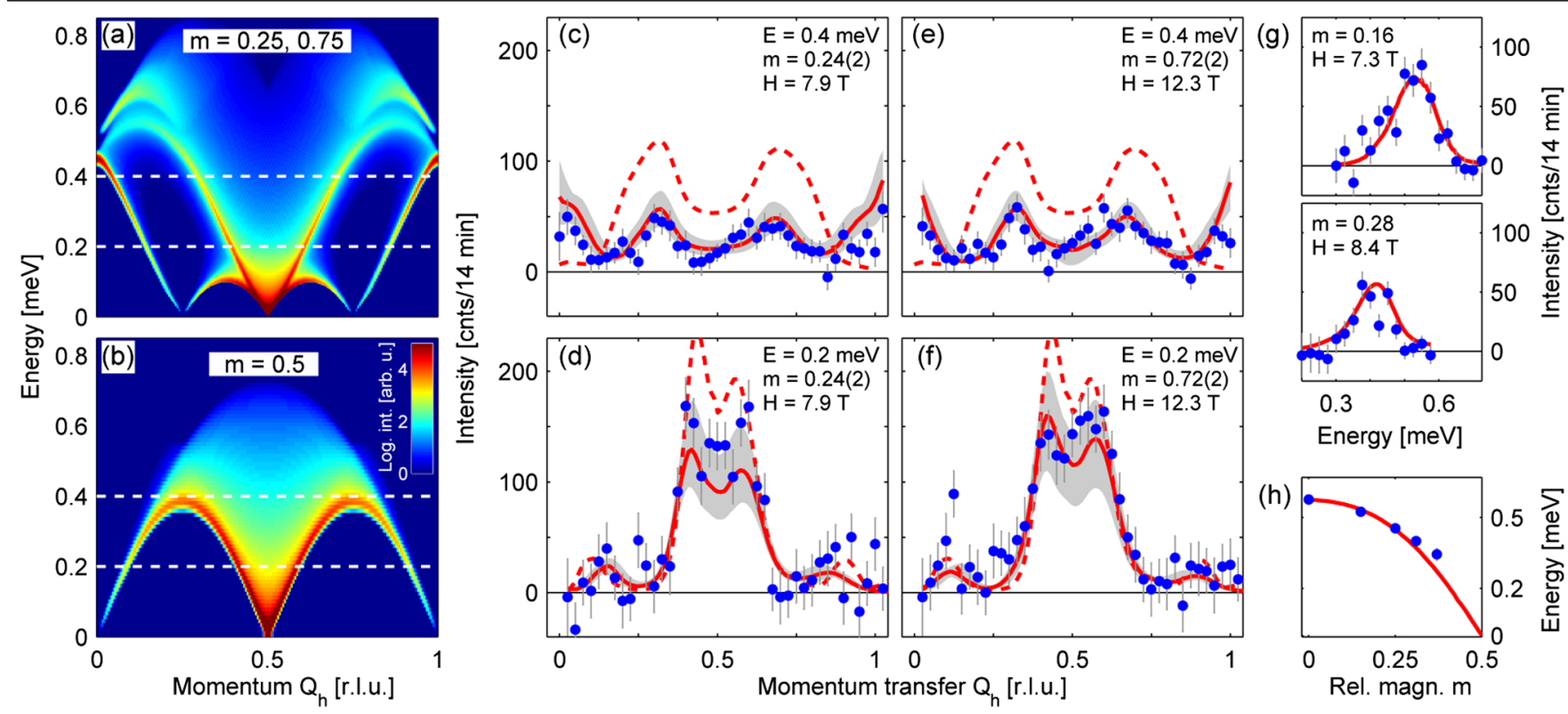

FIG. 3 (color online). Incommensurate excitations in the LL phase. (a),(b) Spinon continua calculated for an $S=1 / 2 X X Z$ chain with anisotropy $\delta=0.5$ (see text) at $m=0.25,0.75$ (a) and $m=0.5$ [(b), data shown also in Fig. 2(a) convolved with instrumental resolution]; dashed white lines represent INS scans at $E=0.2$ and $0.4 \mathrm{meV}$. (c)-(f) Constant- $E$ scans (trajectories as in Fig. 2(b) inset) for $B=7.9$ and $12.3 \mathrm{~T}$. (g) Constant- $Q$ scans at the ZB for two chosen magnetizations $m$ between 0 and 0.5 . (h) Summary of data for ZB excitation energy as a function of $m$. Red lines are predictions based on the $X X Z$ chain: solid from panel (a) and dashed (shown for comparison) from panel (b).

interladder coupling $\left(T_{N, \max }=110 \mathrm{mK},[12,13]\right)$. Second, the spinon continuum studied in Figs. 2 and 3 arises only from fractionalization of the lowest triplet branch of the QD phase.

We observe a continuous evolution of the excitation spectrum as the magnetic field is tuned away from $m=$ 0.5 . Figures 3(c)-3(f) show constant- $E$ scans at $B=7.9$ and $12.3 \mathrm{~T}$ (corresponding approximately to $m=0.25$ and 0.75 ) which are essentially identical within the experimental error. At $E=0.4 \mathrm{meV}$, the decrease of magnetic intensity compared to Fig. 2(b) between $Q_{h}=0.2$ and 0.8 is accompanied by additional weight around the zone boundaries (ZBs, which we define as $Q_{h}=0$ and 1). For these values of $m$, field-induced shifts in spectral weight are less pronounced at $E=0.2 \mathrm{meV}$. Because it is not possible by INS to follow the location of the zeroenergy incommensurate point, instead we have measured the magnetic signal at the $\mathrm{ZB}$ as a function of field [Figs. 3(g) and 3(h)], finding an increase in energy and intensity from $m=0.5$ to $m=0$ and 1 .

When the magnetic field is increased beyond a second QCP at $B_{s}$, the spins are fully aligned. The spectrum becomes discrete again, as shown in Figs. 4(d) and 4(e), with elementary magnon excitations. We observe a 1D dispersive band [Figs. 4(b) and 4(c)], whose width is very similar to that measured in the QD phase. The $\mathrm{ZB}$ excitation energy increases linearly with applied field [Fig. 4(a)], which allows the identification of $B_{s}$.
We use the magnon dispersion relations to deduce the exchange parameters of the system. Because $\left(\mathrm{C}_{5} \mathrm{H}_{12} \mathrm{~N}\right)_{2} \mathrm{CuBr}_{4}$ is rather "strongly coupled" $\left(J_{r} / J_{l} \approx\right.$ 4), high-order perturbative expansions are very effective, and here we follow the (3D) treatment of Ref. [17]. The measured triplet dispersion is dominated by the ladder terms: we obtain $J_{r}=12.8(1) \mathrm{K}$ and $J_{l}=3.2(1) \mathrm{K}$ [black line in Fig. 1(b)]. From the fits to the linear Zeeman splitting [solid lines in Fig. 1(f)], the $g$ factor for this orientation is $g=2.17(3)$, while $B_{c}=6.8(1) \mathrm{T}$. The two approaches agree perfectly, and are consistent with values of $g$ and $B_{c}$ determined by other techniques.

Theoretically, the excitations of a field-polarized ladder are gapped spin waves with dispersion relation $\epsilon\left(Q_{h}\right)=$ $g \mu_{B}\left(B-B_{s}\right)+J_{l}\left[1+\cos \left(2 \pi Q_{h}\right)\right]$ [18]. A mean-field treatment is exact here because all quantum fluctuations are quenched. The red lines in Figs. 4(b) and 4(c) are fitted using this expression: when the small $3 \mathrm{D}$ coupling term is removed, we obtain $J_{r}=13.1(1) \mathrm{K}, J_{l}=3.3(1) \mathrm{K}$, and $B_{s}=13.6(1) \mathrm{T}$. The fit to the ZB energy in Fig. 4(a) yields $B_{s}=13.6(2) \mathrm{T}$.

The LL continuum arises from the fractionalization of spin-flip excitations $\left(\Delta S^{z}=1\right)$ into two elementary and deconfined $S=1 / 2$ objects. Indeed, in a unified description of the full phase diagram, the magnon excitations in the QD and FM phases are bound states of these spinons, and the QCP at $B_{c}$ may be regarded as a field-driven spinon binding-unbinding or confinement-deconfinement transi- 


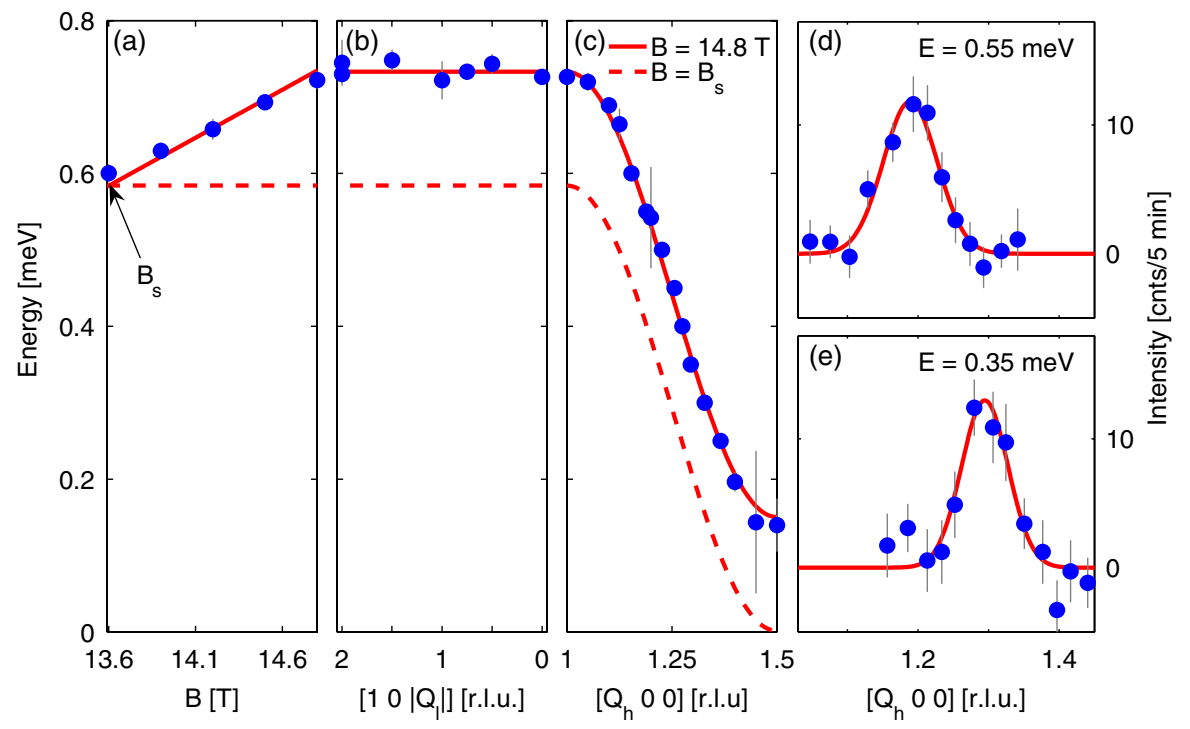

FIG. 4 (color online). Spin dynamics in the FM phase. (a) Magnetic-field dependence of the ZB excitation energy. (b),(c) Magnon dispersions along $Q_{l}$ and $Q_{h}$. (d),(e) Constant- $E$ scans at $B=$ $14.8 \mathrm{~T}$ and $T=50 \mathrm{mK}$ with Gaussian fits. Solid lines in panels (a)-(c) are fits to the dispersion, while dashed lines allow extraction of $B_{s}$. tion. The theoretical description for a ladder in the strongcoupling limit may be obtained by a mapping to the $S=$ $1 / 2 X X Z$ chain with anisotropy $\delta=1 / 2$ [19]. The lowenergy sector of the ladder [i.e., the lowest Zeeman-split branch in Fig. 1(f)] is governed by

$$
\mathcal{H}_{X X Z}=\sum_{i} J_{l}\left(\tilde{S}_{i}^{x} \tilde{S}_{i+1}^{x}+\tilde{S}_{i}^{y} \tilde{S}_{i+1}^{y}+\delta \tilde{S}_{i}^{z} \tilde{S}_{i+1}^{z}\right)-b_{\text {eff }} \tilde{S}_{i}^{z},
$$

where the effective field $b_{\text {eff }}=2 b_{s} \frac{B-\left(B_{c}+B_{s}\right) / 2}{B_{s}-B_{c}}$ is such that $-b_{s} \leq b_{\text {eff }} \leq b_{s}$, with $b_{s}=\frac{3}{2} J_{l}$ the saturation field for the $X X Z$ chain. This effective model has spinon excitations in whose dispersion $J=J_{l}$ and $\alpha=1.299$ [16], whence the bounds $\epsilon_{l}\left(Q_{h}\right)$ and $\epsilon_{u}\left(Q_{h}\right)$ shown as solid lines in Fig. 2(a). Because $\tilde{\boldsymbol{S}}_{i}$ in Eq. (1) is a composite of the two physical spins on each ladder rung, the total INS cross section contains a rung structure factor modulating the contributions from longitudinal and transverse spin correlations [14]. All data in the LL phase were measured on the maxima of the transverse structure factor (insets in Fig. 2), where the longitudinal contributions are zero.

We have calculated the transverse spin correlation function for all values of $m$ following Ref. [20], and in Figs. 3(a) and 3(b) present the results for $m=0.25,0.75$, and 0.5 . In the $X X Z$-chain model, the spectral intensities are symmetric in $m$ about $m=0.5$. Data taken at $B=$ $10.1 \mathrm{~T}$ correspond to $m=0.48(2)$, while fields $B=7.9$ and $12.3 \mathrm{~T}$ correspond, respectively, to $m=0.24(2)$ and $0.72(2)$. The theoretical intensities were convolved with the 4D instrumental resolution to obtain the global fit shown as red lines in Figs. 2(b)-2(d) and 3(c)-3(f), while the red lines in Figs. 3(g) and 3(h) are obtained directly. The shaded bands indicate the error bar in the experimental determination of a single constant of proportionality valid for all fields, energies, and wave vectors. Their width combines the statistics of all our scans with uncertainties in the exact magnetization values at the chosen fields and in the convolution procedure. The agreement is quantitatively excellent. We note, in particular, that only the smallest asymmetries between $m<0.5$ and $m>0.5$ may be discerned in the data [comparing Fig. 3(c) with 3(e) and Fig. 3(d) with 3(f)], and that the energy dependence of the intensity is described exactly [Fig. 2(c)].

Measurements in the QD and FM phases provide two independent and complementary determinations of $J_{r}$ and $J_{l}$. While the values of $B_{c}$ and $B_{s}$ deduced from these are marginally smaller than from thermodynamic studies, the latter were performed mostly on undeuterated samples and in different orientations. By exploiting the $\boldsymbol{Q}$ specificity of INS, we have accounted for a $3 \%$ effect from interladder coupling [14]. We then find that the intrinsic ladder leg parameters in the two regimes are identical within their errors, but the rung parameters are not: this apparent magnetostriction effect is of order $1 \%-2 \%$. That such a phenomenon may occur is not surprising in a structurally "soft" material of this nature [10]. In fact this discrepancy is the sum of all additional contributions, including any other magnetoelastic terms or complex spin interactions. Our magnon dispersion analysis therefore quantifies the statement that $\left(\mathrm{C}_{5} \mathrm{H}_{12} \mathrm{~N}\right)_{2} \mathrm{CuBr}_{4}$ is an excellent spin-ladder system.

A key property of the LL spectrum is the presence of a zero-energy mode at an incommensurate wave vector $0<$ $Q_{\min }<1 / 2$ which changes systematically with field [21]. However, the spectral weight at $Q_{\min }$ vanishes as $E \rightarrow 0$ [Fig. 3(a)], precluding a direct measurement of the incommensurability. Instead we have presented indirect confirmation of the theoretical prediction in the form of the fieldtuned finite- $E$ spectra and the ZB energy [Figs. 2 and 3]. The question of the evolution of spectral weight in the LL is of particular interest in the context of the commensurate 3D ordered phase which emerges at sufficiently low temperature $[12,13]$. At intermediate energies, we return to the question of the symmetry of the measured spectra about 
$m=0.5$ : physical effects arising due to departures from strict strong coupling, and from the higher triplet branches, are expected to cause some asymmetry in intensities, but these are clearly extremely small for energies $0.5 J_{l}<E<$ $2.5 J_{l}$. At high energies, it remains to address, both experimentally and theoretically, the nature of the higher spinon continua expected from the upper two triplet branches.

In summary, we have performed a comprehensive INS investigation of the magnetic excitation spectrum in $\left(\mathrm{C}_{5} \mathrm{H}_{12} \mathrm{~N}\right)_{2} \mathrm{CuBr}_{4}$, a spin-ladder compound whose energy scales are perfectly suited to systematic studies in laboratory fields. We observe the presence of a broad continuum of spinon excitations in the intermediate, Luttinger-liquid (LL) phase, which is starkly different from the discrete (magnon) excitations measured below the critical field (QD phase) and above saturation (FM phase). From the QD and FM results, we extract the ladder parameters with unprecedented accuracy, demonstrating directly that even the sum of all other effects beyond the "nearly ideal" Hamiltonian of weakly coupled ladders falls below the $2 \%$ level. The spinon continuum proves the occurrence of field-induced fractionalization into elementary $S=$ $1 / 2$ entities as the system enters the LL regime. An excellent, fully quantitative description of the incommensurate continua measured at all fields is obtained from an effective chain model for a ladder with the coupling ratio $J_{r} / J_{l} \approx 4$ of $\left(\mathrm{C}_{5} \mathrm{H}_{12} \mathrm{~N}\right)_{2} \mathrm{CuBr}_{4}$.

This project was supported by the Swiss National Science Foundation (Division II and the NCCR MaNEP), the Royal Society, EPSRC, and FOM. This work is based partially on experiments performed at the Swiss spallation neutron source, SINQ, at the Paul Scherrer Institute.

[1] T. Giamarchi, C. Rüegg, and O. Tchernyshyov, Nature Phys. 4, 198 (2008).

[2] A. Zheludev et al., Phys. Rev. Lett. 88, 077206 (2002).

[3] V. O. Garlea et al., Phys. Rev. Lett. 98, 167202 (2007).

[4] M. Clémancey et al., Phys. Rev. Lett. 97, 167204 (2006).

[5] D. C. Dender et al., Phys. Rev. Lett. 79, 1750 (1997).

[6] M. B. Stone et al., Phys. Rev. Lett. 91, 037205 (2003).

[7] B. Lake et al., Nature Mater. 4, 329 (2005).

[8] B. R. Patyal, B. L. Scott, and R. D. Willett, Phys. Rev. B 41, 1657 (1990).

[9] B. C. Watson et al., Phys. Rev. Lett. 86, 5168 (2001).

[10] T. Lorenz et al., Phys. Rev. Lett. 100, 067208 (2008).

[11] Ch. Rüegg et al., Phys. Rev. Lett. 101, 247202 (2008).

[12] B. Thielemann et al., Phys. Rev. B 79, 020408(R) (2009).

[13] M. Klanjšek et al., Phys. Rev. Lett. 101, 137207 (2008).

[14] B. Thielemann et al. (unpublished).

[15] L. D. Faddeev and L. A. Takhtajan, Phys. Lett. 85A, 375 (1981).

[16] G. Müller et al., J. Phys. C 14, 3399 (1981).

[17] M. Müller and H.-J. Mikeska, J. Phys. Condens. Matter 12, 7633 (2000).

[18] B. Normand, Acta Phys. Pol. B 31, 3005 (2000).

[19] F. Mila, Eur. Phys. J. B 6, 201 (1998).

[20] J.-S. Caux, R. Hagemans, and J. M. Maillet, J. Stat. Mech. (2005) P09003.

[21] R. Chitra and T. Giamarchi, Phys. Rev. B 55, 5816 (1997). 